\title{
When Code Governs Community
}

\author{
Yubo Kou \\ Purdue University \\ $\underline{\text { kou2@purdue.edu }}$
}

\author{
Xinning Gui \\ University of California, Irvine \\ guix@uci.edu
}

\begin{abstract}
We present a qualitative study of governance in the community of League of Legends, a popular Multiplayer online battle arena (MOBA) game developed by Riot Games. To cope with toxic behaviors such as griefing and flaming, Riot Games initially implemented a crowdsourcing system inviting players to participate in governing their own community. However, in May, 2014, they automated the system, relying heavily on code while minimizing the level of human participation. We analyzed both players' and Riot Games' narratives to understand their attitudes towards the relationship between human judgment and automation, as well as between alienation and community. We found stark differences between players and Riot Games in terms of attitudes towards code and value in designing online governance. We discuss how the design of governance might impact online community.
\end{abstract}

\section{Introduction}

The prevalent toxicity across video games and online communities has posed both theoretical and practical challenges to governance $[1,12,21,38]$. What constitutes the optimal mode of governance, as well as under what criteria $[7,9,13,22]$ has always been a contested topic, with no definite answer.

Recent years among the heated debate about governance rise two prominent research strands, one stressing the value of human judgment [36,39] while the other recognizing the power of code, or algorithms $[11,26]$. These two ideas seem to occupy the two ends of a continuum [2], suggesting deep-seated tensions that impact both online communities and their owners.

We explore the tension between human and code, as well as how they are factored into online governance. Our site is League of Legends (LoL), a popular Multiplayer online battle arena (MOBA) game developed by Riot Games. In the past two years, the League of Legends community's governance mode has undergone drastic changes. Between May, 2011, and April, 2014, Riot Games implemented and maintained the "Tribunal System," which used crowdsourcing to invite players to judge and punish toxic players [21,22]. Since April, 2014, the system has undergone maintenance and implemented an automated system that still operates today. The League of Legends community thus experienced two strikingly different governance modes.

We analyzed player forum discussions during these two phases, as well as Riot Games' official announcements. We found that players and Riot Games differed a lot in their attitudes towards governance. While the game company stressed the efficiency, sophistication, and robustness of code, players expressed deep concerns regarding the limitations of code. The sharp opposition between human judgment and automation in this study raises critical questions regarding the relation of governance to community.

\section{Related Work}

In this paper, we use toxic behavior and deviant behavior interchangeably. Toxic behavior refers to "conduct that departs significantly from the norms set for people in their social statuses" [28]. Game scholars have studied toxic behaviors such as flaming [10,21], trolling [30,37], cheating [4,17], and griefing [7,12,24]. Psychologists found that toxic behaviors are prevalent in online games due to online psychological effects such as anonymity and de-individuation [3,6,18,34]. Such behavior is considered detrimental to player experience as well as game community.

What constitutes toxicity in a particular social setting is related to its norms. However, player norms are often contested and evolving [35]. Humphreys and de Zwart demonstrated that player norms often differ from rules such as game mechanics, TOS, and EULA [14]. Johansson and Verhagen found both differences and similarities of player-made rules across different clubs in online games [15].

Therefore, it is often challenging to determine what constitutes toxic behavior. For example, Foo and Koivisto found that certain play style was allowed in one game, but forbidden in another, and players might 
unintentionally disrupt others' experience [8]. Lin and Sun reported that adult players sometimes created a griefer stigma for adolescent players to relive their anxiety, further complicating the common perception of grief [24].

Players familiar with the changing norms can play an important role in coping with toxic behaviors. Learning from the experience with running Habitat, one of the first multi-player online virtual environments, Morningstar and Farmer argued that "a virtual world need not be set up with a 'default' government, but can instead evolve one as needed" [29]. Foo documented player involvement in grief play management at varied degrees across different game platforms [7]. In competitive and intense games like League of Legends, ranking plays an important role in player experience [20]. In order to win a match, players might develop a more proactive culture towards deviant behavior [19].

\section{Background}

League of Legends (LoL) is a free-to-play matchbased game. A match takes place between two teams, each of which contains five players. When a player queues for a match, they are randomly matched with four teammates, as well as an opponent team. Each player needs to choose a role as well as a champion. At the time of writing this paper, LoL has more than 130 champions, and each has a unique set of abilities. After champion selection, two teams enter a match and start from a square map's lower-left and upper-right corner. A team wins the game by destroying the other's base or forcing the other to surrender (see Figure 1).

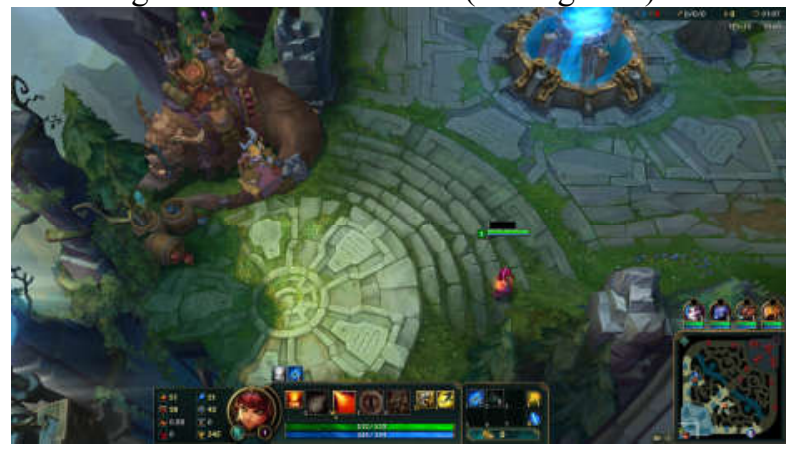

Figure 1. A Match in League of Legends.

Since the release of League of Legends, Riot Games had received tens of thousands of player complaints about toxic behavior [33]. The severity of toxic behaviors ranges from AFK ("away from keyboard") and flaming to sexual harassment, personal attack, and racist language. To cope with this problem, Riot Games devised the Tribunal System in May, 2011 .
The Tribunal worked as follows: First, players could report deviant players immediately after a match. The Tribunal generated a case (see Figure 2) if a player was reported frequently enough. Second, players could log onto the Tribunal and judge cases. The Tribunal allowed players to judge with no compensation most of the time. We use "judge" to refer to players who judged cases in the Tribunal.

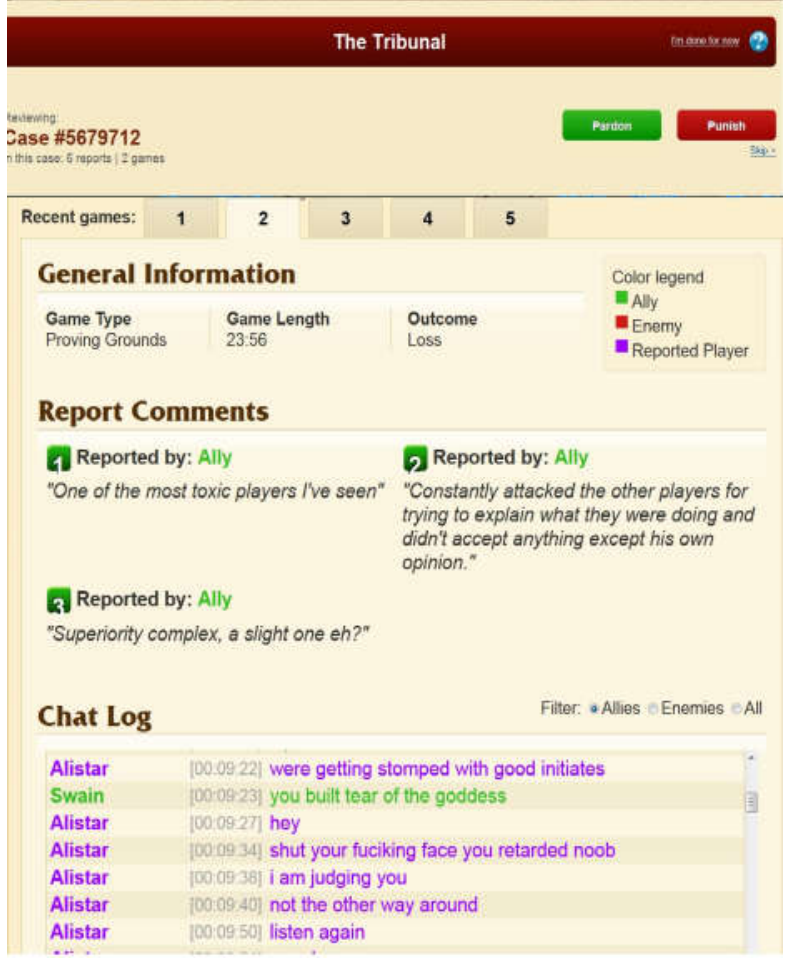

Figure 2. A Tribunal Case.

A Tribunal case was reviewed by multiple judges (Riot Games has not disclosed the exact number of judges needed for a case). Judges could not discuss or communicate in the Tribunal. The Tribunal assigned punishment if the majority of the judges agreed. The Tribunal punished a deviant player using an account suspension. The punished player would receive a link to his case, which Riot Games called "reform card."

Riot Games also built a "justice review" feature into the Tribunal system (see Figure 3). The feature presented to a judge his or her judging history, including the total cases reviewed, accuracy, justice rating, ranking, a list of all the previous cases, etc. The Tribunal determined the judgment as "correct" if a judge's decision agreed with the majority vote. Accuracy was calculated based on the percentage of cases that a judge made the "correct" vote. The justice rating was calculated based on a judge's total cases reviewed and accuracy. The ranking was determined by the judge's justice rating among all the judges. 


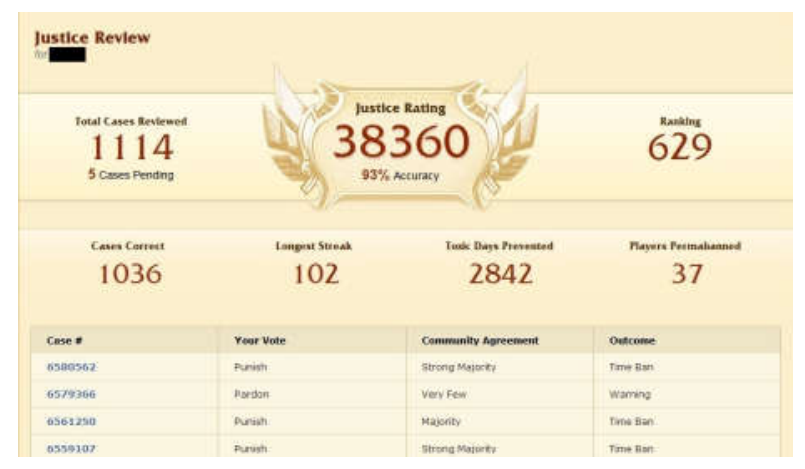

Figure 3. Justice Review in the Tribunal.

The Tribunal system helped the formation of what Kou and Nardi consider as a hybrid system of governance [22], where players and Riot Games work together to govern the LoL community. During the functioning period of the Tribunal, players engaged in interpreting Riot Games' ambiguous rules, the "Summoner's Code," as well as articulating player norms.

Besides the Tribunal, Riot Games has also deployed a wide range of techniques to promote acceptable player behavior. For example, players can "honor" their teammates or opponents with badges including "helpful," "teamwork," "friendly," and "honorable opponent." The game also shows messages that encourage teamwork during its loading screen, such as "Teammates perform worse if you harass them after a mistake."

\section{Methods}

This study belongs to a larger project that uses ethnographic methods to understand governance in the League of Legends community. The first author started playing the game in September, 2011. He also judged over 1000 cases in the Tribunal. In this paper, we present two sets of data. The first includes 320 forum threads in which players discussed governance in League of Legends. The second contains thirteen official announcements from Riot Games about the Tribunal, which can be found on LoL's website. ${ }^{1}$ We adopt a grounded theory approach [32] to analyze collected data. In section 5 and section 6 , we will detail our data collection strategy and data analysis process.

\section{Analysis of Player Discussion}

We searched "Tribunal" in both the old official forum and the new official forum for LoL, and

\footnotetext{
${ }^{1}$ http://na.leagueoflegends.com/en/news/game-updates/playerbehavior
}

collected 161 forum threads that took place during the human-judged era, and 159 forum thread that happened during the era of automation.

To analyze these forum threads, we first read the data and used our initial perceptions to generate a starting list of codes. These included ideas about players' different opinions about human judgment and code judgment. From there, we returned to the data to conduct a systematic analysis of the themes that arose. After several iterations of coding, we identified a central phenomenon in the data - the major elements of governance that concerned players. With this frame in mind, we returned to the data to find episodes where players talked about these elements.

We divide our findings into two sections. The first section reports how players talked about governance in the era of Human Judgment, and the second section reports how players thought about governance during the era of automation.

\subsection{The Era of Human Judgment}

In this section, we report the themes in player discussions regarding human-judged Tribunal cases. We found five themes out of the 161 forum threads.

Understanding the Tribunal. We have 77 threads discussing the Tribunal's mechanisms. The Tribunal was a "black box" to LoL players. Neither judges or non-judge players knew the mechanisms and statistics behind the Tribunal. LoL players thus accumulated knowledge about the system through forum discussions. For example, below is an excerpt from a post:

Facts behind the Tribunal (A guide with math)!

Hello fellow Summoners!

I have been a big fan of the Tribunal since I began playing League of Legends. I was dying to see the math behind it and provide a guide on the math behind the tribunal. I achieved this guide by reading every post by Lyte I could find over the past 3 months. I hope you find it as informative as it is interesting. Please sticky!

In this thread, the player compiled a list of facts about the Tribunal from news reports and the official announcements for his fellow players to read.

In a significant portion of these threads, we noticed the active participation of Riot employees. They explained how the Tribunal worked and instructed players in its use. For example, here is an excerpt from a forum thread started by Riot employee Lyte with the title "Lead Social Systems Designer:"

Lately, I've been receiving a lot of e-mails about the same questions and complaints about the Tribunal. 
I'm working with WookieCookie, RiotSeb and statuskwoh to add these to the Tribunal FAQs but we would love to discuss them here first.

1) What's the point of having a language filter if I can get banned for 'adult' language?

a) We aren't out to punish players who use adult language; in fact, very few players are ever banned for saying stuff like "f***, I missed that skill shot" or "damn, f***ing close game!" However, the language filter is not an excuse to verbally abuse someone in the game. When you verbally abuse or harass someone by calling them " $f * * * i n g * * * * * *$ " or " $n *$ gger noob", that's when you've crossed the line and will be punished by the Tribunal. I've said this before and I'll say it again, we aren't trying to clean online games of adult language; we're trying to reform or get rid of jerks who like to verbally abuse and harass other players.

Riot employees' involvement in forum discussion disseminated expert knowledge and opinions to ordinary LoL players, and oftentimes spurred more player participation.

Questioning the Tribunal. The forum did not lack heated debates regarding the legitimacy and reliability of the Tribunal; 24 threads questioned the Tribunal. Players doubted the efficiency of the Tribunal, as well as the responsibility of the Tribunal judges. In this excerpt, a player expressed his doubt in an emotional manner:

This system is a joke. Sure, riot reviews cases before they hand out bans, but why can't they stop being lazy and just have a team for doing this.

A large majority (20) of these threads led to heated debates, attracting a large number of LoL players either defending or attacking the Tribunal. For example, in response to the above comment, we observed players' thoughts about how the Tribunal was necessary. Here are a few examples where players supported the Tribunal:

Episode 1: I'm ok with this system because I cannot come up with something better for a f2p 35 million playerbase game without the company losing time + money to create anti-troll measures

Episode 2: So let me get this straight. You're angry with a system that's designed to help prevent the innocent from being punished by providing information from the game, because someone who you saw shouldn't be punished and the system you're angry about provided evidence as such?

What am I missing?

We also have examples where players opposed the Tribunal:

Episode 1: I'm saying there's a system that shouldn't have the option for these people to be banned, for instance, if someone was dumb enough to think what they did was a bannable offense.

Episode 2: The problem is that the case made it to tribunal at all. And that the vast majority of people are stupid. Therefore, if enough people are participating in the tribunal thing, then there's a good chance there's a bunch of idiots more than willing to just straight punish them.

Understanding Behavioral Issues. In the official forum, Tribunal judges could discuss behavioral issues and punished players could share their case for fellow players to comment on. We collected 31 threads where players discussed specific behaviors and reached mutual understanding of what constituted toxic or nontoxic behavior. For instance:

I really want to know if this was deserved, since most of these reports are just duo queues who don't like me in ranked it appears... Like I get that game 2 was bad, but everything else I get the **** flamed out of me then reported for not really doing anything in my opinion. Game 3 I probably shouldn't have argued matchups, but I was just giving my opinion since if I give a lane FB, I expect them to be able to do something with it aside from proceed to feed...

http://na.leagueoflegends.com/tribunal/en/case/657 $\underline{83471}$

Sometimes Riot employees started threads to openly discuss certain cases. For example, Pendragon, titled "Director of Player Experience," started a thread to ask the community to collectively judge a case:

Greetings,

Something that we've wanted to experiment with is the idea of having more public Tribunal cases where we can have an open dialog with the community about the details of a specific case and why it might have gone the way it did... on occasion players decide to opt-in to public conversations about their Tribunal cases, including this recent example: http://na.leagueoflegends.com/board/sho wthread.php? $t=2243272$ The arrangement we made is that if the Community believes he deserves to be punished he has to write individual apology letters to every person on his team in each of the games in his recent Tribunal case. If the Community does not believe he deserves to be punished then his suspension will be lifted. It's up to you decide whether or not to uphold the Tribunal verdict or overturn it. We'll use your discussion and feedback in this thread to help guide future improvements to the Tribunal and behavior handling. 
The thread garnered 6961 player votes, and a vast majority (5813) voted to punish the player.

Debating the Design. Players discussed many features of the system in 16 threads. They debated better ways to improve the Tribunal's efficacy. Here is an example:

I believe the Pre-game chat should be included in the Tribunal cases as that is what causes a lot of bad games, especially in ranked. To be able to judge the player as fairly as possible, the pre-game chat is where, I believe, about $50 \%$ of bad behavior begin.

Seeking Fun from the Tribunal. Players chatted about the Tribunal in a playful way. We found 13 threads where they exchanged fun quotes they found in the Tribunal cases, or made jokes about the Tribunal. Here is an example:

Post your favorites quotes you found until now. It can be from the person who reported, the culprit himself, or the other players.

From my three cases today:

"Vulgar display of character, every perspective of this mans life must be in disgusting dismay, he is the most offensive mouthed player $i$ have ever seen in all my time playing league, and he has no team effort at all, please for the love of all that is good, if you read any of these reports, read this one. I wish $i$ could choose 12 of these select offenses but $i$ cant."

"Tristana is terrible solo nubs. GG im afk."

"You dumb amaricans can't even spel correctly lolololol"

The threads from the era of human judgment indicate a participatory governance culture in which players engaged in learning the Tribunal, figuring out how to use or improve the Tribunal, and deepening understanding of player norms. Doubts expressed against the Tribunal often attracted a large amount of replies, in which players debated in a serious, constructive manner.

\subsection{The Era of Automation}

In this section, we report three themes that emerged out of our coding of 159 threads that took place during the era of automation.

Bringing the Old Tribunal Back. In 117 threads, players voiced the request that Riot Games should again allow players to judge the Tribunal cases. They were discontent with the automated way to judge their cases.
Tribunal: Are we getting our power back Riot?

So, I want to start off with this: The Tribunal gave the players a power that most communities won't have, and that was the power to moderate ourselves. We were our own moderator, and the tribunal was even essential in banning pros for their toxicity. They've changed since their bans, but there used to be a due process for people getting banned, and the players were an intimate part of that for the longest time.

This is where I'll ask: Are we even going to see the tribunal ever again?

Inquiring about the Status Quo. Players who did not frequently check updates to the Tribunal were surprised to see that the old Tribunal was taken down. We have 40 threads in which they asked questions regarding how the new Tribunal worked. For example, a player asked this question:

Ok I have a question.. I've been trying to get on it for maybe the past week or even more. It keep saying it looking for more cases, but it never seems to have any. Nothing ever come up or is shown each time I try or refresh it. Is it down or still being worked on for the upcoming remaking? Some kinda notice of what up would be nice. I'm a fan of making this game a better place to be.

Discussing the Design. Two players proposed design suggestions for the Tribunal, drawing upon their own experience to discuss what could be done to improve the current system. For example, a player wrote:

Needed Revisions to Tribunal Awarding of AFK Timers and Low Priority Queues

The League of Legends penalty system for leaving/AFKing games has always been severe, which is a good thing! This encourages players to not AFK, and prevents a lot of frustration (we've all been there, stuck in a $4 v 5$ waiting for 20 minutes).

But I think RIOT needs to change how they give them out.

The threads from this era reveal a general feeling of dissatisfaction among players. Players reminisced about the "good old times," when they could participate in governing their own community. In the next section, we will analyze why players favored human over code in the Tribunal.

\subsection{Comparing Human judgment and Automation}

In this section, we looked closely at why players disliked automation and preferred human judgment in 
the Tribunal. We found three recurring themes in the 117 threads where players compared human and code judgments.

Inability to understand context. Players argued that code could only detect toxic language at the word level, but was unable to decipher the particular context in which toxic terms were used. However, human conversation was highly contextual and nuanced. What constituted toxic in one context might not be the case in another. For example, a player wrote:

Bring back the tribunal

Automated systems can't accurately assess complex human interactions.

You do not have an AI capable of this. I don't care how good you think you are at programming, you don't. It may be able to tag people who are overly toxic for no good reason, but outside of those instances it is not effective at accurately assessing human interactions.

Why not keep your special little automated system to detect the worst offenders, and bring back the tribunal for every thing else? Or at least funnel the auto mated bans through the tribunal so that actual people can assess the situations.

But of course, this would only happen if Lyte had a soul. Lyte is a soulless foot stool.

Another player wrote:

In short; it's ineffective and abused; the ai is just not capable of understanding context nor can it read between the lines, but it'll punish anyone targeted by several reports. Which leads to cases where people repeatedly get setup with the same troll, and other cases where a premade troll team will all just report the same person. These combined problems have drastically increased toxicity, and even made people permanently quit the game.

Opaqueness. Players complained about the opaqueness of the new Tribunal. They felt they were receiving less information about the system when code governed the community. For example, a player wrote:

What ever happen to the tribunal? Riot just takes it away, then decides never to bring it back? but leaving it up like it will come back? We don't get to be involved like we use to, riot is straying away from the community and becoming all about themselves. but meh, its ok, because s a bitch ;)

Another player wrote:

I'm not sure how the automated system works. It seems like it is keyword banning and/or automated punishments being issued without human review. The reform cards are suppose to release some time this month. However, if you aren't allowed to see the reform card of people you are matched with, then I feel this system will be exploited or again unsuccessful.

Vulnerability. Players discussed how code was vulnerable to the manipulation of toxic players. They were concerned that toxic players could easily game the system to avoid punishments. For example, a player wrote:

Don't say stuff in in game chat or post game lobby that makes you look toxic. Its incredibly easy. I would not spend money on this game because I think the automated ban system is unfair and this ban system can pretty much screw you out of the money you spent with no repercussions.

Another player wrote:

It was slow, but effective, with the computerized system, $u$ can troll 100s of games so long as $u$ remain silent the whole time.

Our analysis of players' discussions shows that players gave serious thought to the disadvantages of automation. In their accounts, humans outperformed code in judging cases, and were more reliable.

\section{Analysis of Riot Games Announcements}

We collected thirteen official announcements from LoL's website's "player behavior" category. The dates of these announcements span from September, 2013, to May, 2016. However, the majority (12) of these announcements took place after the Tribunal transitioned into the era of automation, and the remaining one is an official video promoting teamwork.

To analyze these official announcements, we first read the data and tried to generate codes about how Riot Games talked about governance in LoL. We paid particular attention to Riot Games' design principles for the Tribunal. From there, we returned to the data to conduct a systematic analysis of the themes that arose. After several iterations of coding, we identified three principles that these announcements often refer to. With this frame in mind, we returned to the data to find relevant episodes.

Instant decisions. These announcements repeatedly highlighted how code can make instant decisions to punish toxic players, suggesting that Riot Games values the efficiency of code over human. For example, this excerpt is from the announcement explaining why the human component would be removed: 
We're going to temporarily put the Tribunal in maintenance mode while we upgrade the overall system to ensure players who deserve punishments receive them much faster than they currently do...In the meantime, a new system will hand out restricted chat punishments and game bans rapidly so players will be banned or placed into restricted chat mode within a few hours of their negative behavior.

In two additional announcements, Riot stated that instant decisions could more effectively improve player behavior:

Episode 1: we're seeing this type of language in less than 3\% of games globally and when it does appear, it's immediately reported by players and acted upon.

Episode 2: Reform becomes increasingly likely the shorter the feedback loop (the time between the negative behavior and receiving feedback that the behavior was not acceptable).

Sophisticated Code. Riot Games stressed how advanced and sophisticated code was in making the correct decisions. Here is an example:

With ranked restrictions, we used an advanced machine-learning model to instantly recognize negative behaviors the community rejects. Through end-of-game reports, honors and historical Tribunal records, we were able to analyze millions of games in order to identify negative behaviors and build a faster feedback loop.

Effective psychological measures. Riot Games often cited psychological principles when explaining mechanisms behind its code. Here is an example:

One of the keys of positive reinforcement is the idea of "schedules," or the expected frequency of a reinforcing event. Introducing surprise rewards unrelated to specific activities or durations is one of the most effective ways to encourage positive player behavior. The surprise element is crucial: imagine an achievement system where, if you are sportsmanlike for your next 10 games, you unlock a free skin. Players could simply behave for 10 games, unlock their gift and go back to playing the same way they were before (whether that's positive, negative or neutral). So, instead, we'll continue to surprise players once in a while for their positive behavior. Because players aren't sure what the next reward is (or when it is), players will strive to be sportsmanlike in a larger range of games to try to get all the surprises.

Such value is also evident in the Tribunal designer Jeffrey Lin's talk "the science behind shaping player behavior in online games" [25].

\section{Discussion}

In this paper we reported an analysis of player discourses as well as official narratives with regard to a major shift in governance in the League of Legends community. Players showed different levels of engagement in these two eras. During the first era, Riot Games engaged in frequent communication with the community to clarify misunderstandings and encourage players to participate. Players actively discussed and participated in the Tribunal. Even their negative comments against the Tribunal displayed careful thinking and sound rationale. Johansson et al. also found that LoL players had more trust in the Tribunal system, compared to DOTA 2 players who discussed the system [16]. In the era of automation, much less communication was observed between Riot Games and the community. Players showed distrust in automated method, perceiving it as an ineffective, mechanistic tool.

We show how the values of players versus Riot seemed to converge during the human-judged era, when they worked together to improve the Tribunal's efficiency. We also show how their values began to diverge as League of Legends entered the era of automation. As per our analysis of Riot Games' announcements, the game company's accounts of their code manifested the modernist values elaborated by Ritzer [31]: efficiency, predictability, calculability, substitution of non-human for human technology, and control over uncertainty. These values stressed finding the best means to any given end, ensuring that the result is predictable, quantifying human behavior, rationalizing the role of humans, and controlling both the certainties and uncertainties of human behavior. Both of the governance modes reflect Riot Games' modernist approaches. Even in the era of human judgment, Riot Games designed the Tribunal in a way that minimizes the task of humans to make judgments by providing them with limited in-game information and allowing them to choose only one out of the three options (pardon, punish, or skip). In sharp contrast, LoL players embraced individualistic values such as participation, autonomy, competence, and relatedness. They reminisced about the era of human judgment even though their then power was rather limited.

Our analysis of players' and Riot Games' different attitudes signified the long-standing tension between community and its corporate owner. For example, Blizzard Entertainment and the Addon community had deep agreement regarding the ownership of World of Warcraft Addons [23]. In 2015, mass collective action took place on reddit to protest the company's ban of several subreddits for harassment, and forced the resignation of the then reddit CEO [27]. Numerous 
incidents show that the value and interest of users and companies do not always align. Such tension renders governance a thorny issue, as it concerns not only the efficiency of containing toxicity, but also player culture and player community.

Governance has a participatory dimension, acknowledged or not by corporate decisions. Even in spaces clearly, strictly defined by owners, people have agency and creativity to find ways to push the boundary and subvert the existing structure [4]. In the era of human judgment, when Riot Games limited judges' communication to zero in the Tribunal system, they used public forums to communicate judging experience and understanding of the system. The Tribunal also had an impact on many other aspects of the LoL community. For example, players enjoyed reading funny quotes from Tribunal cases. Even in the era of automation, we observed players discussed the design of the Tribunal. Players desired to be involved in the governance process. We suggest that the deprivation of participation might contribute to players' preference of the human judged era over the second era.

However, Riot Games seemed to maintain an instrumental view of governance, focusing upon improving code to constrain toxicity in a fast and precise manner. Such intention partially met players' need for a positive game experience. However, due to the lack of public data with regard to the details of the automated system such as report/punish rate and less communication between Riot and the community regarding the subject matter, players were not easily convinced about its efficiency. Moreover, such view of governance conflicted with the Tribunal's history of participatory governance. We conjecture that without such history, players might be more easily satisfied with an automated system.

Crenshaw and Nardi pointed to the significant value of online games' social affordances [5]. They found that developers often neglected players' social needs and designed systems that valued efficiency at the cost of social experience. As a result, some players went to private servers where they could recreate social experience. In this regard, governance once became an important experience for players during the first era. Players could chat with others about the Tribunal, laughed at funny Tribunal quotes, engaged in alternative ways of participating in the way. The removal of the participatory part did reduce many experiences that players developed around the Tribunal. However, LoL players could not recreate such experience in other venues.

We do not necessarily see human judgment and automation as mutually exclusive. On one hand, even the automated system still needs players to identify toxicity, and press the "report" button and select among a few report reasons. Human participation has been minimized, but not entirely excluded. On the other hand, Tribunal judges were sometimes questioned about their responsibility and expertise. We thus see both approaches with distinct advantages and disadvantages, with the potential to complementing each other. For example, while code gives out binary decisions of either punish or pardon, borderline cases might benefit from human judgment.

\section{Limitations}

Our data collection only covered a limited amount of threads on one forum with players mostly from the North America Server. Therefore, the results might not be generalizable to the whole League of Legends community, which has a massive player population. We also lack information showing player demographics, as such information is not accessible from forum threads. The contribution of the paper lies in deepening our understanding of online governance in light of human judgment and automation.

\section{Conclusion}

In this paper we report an analysis of player accounts as well as corporate narrative of governance in the League of Legends community. By comparing player discussions in the era of human judgment and in the era of automation, we were able to reveal a sharp distinction between the values of players and Riot Games. Code was perceived by players as an ineffective and mechanistic instrument. We caution against treating governance from an instrumental perspective. Governance should be understood in its relation to a myriad of people, organizations, and artifacts.

\section{References}

[1] Alonzo, M. and Aiken, M. Flaming in electronic communication. Decision Support Systems 36, 3 (2004), 205-213.

[2] Bruckman, A., Curtis, P., Figallo, C., and Laurel, B. Approaches to managing deviant behavior in virtual communities. Conference companion on Human factors in computing systems - CHI '94, ACM Press (1994), 183-184. [3] Chen, V.H.-H., Duh, H.B.-L., and Ng, C.W. Players who play to make others cry: the influence of anonymity and immersion. ACM (2009), 341-344.

[4] Consalvo, M. Cheating: gaining advantage in videogames. (2007), 246.

[5] Crenshaw, N. and Nardi, B. "It Was More Than Just the Game, It Was the Community": Social Affordances in Online 
Games. 49th Hawaii International Conference on System Sciences (HICSS), (2016), 3781-3790.

[6] Festinger, L., Pepitone, A., and Newcomb, T. Some consequences of de-individuation in a group. The Journal of Abnormal and Social Psychology 47, 2, Suppl (1952), 382389.

[7] Foo, C.Y. Grief Play Management. VDM Verlag, 2008. [8] Foo, C.Y. and Koivisto, E.M.I. Defining grief play in MMORPGs: player and developer perceptions. Proceedings of the 2004 ACM SIGCHI International Conference on Advances in computer entertainment technology - ACE '04, ACM Press (2004), 245-250.

[9] Forte, A. and Bruckman, A. Scaling Consensus: Increasing Decentralization in Wikipedia Governance. IEEE Computer Society (2008), 157-.

[10] Fox, J. and Tang, W.Y. Sexism in online video games: The role of conformity to masculine norms and social dominance orientation. Computers in Human Behavior 33, (2014), 314-320.

[11] Gillespie, T. The Relevance of Algorithms. In T. Gillespie, P. Boczkowski and K. Foot, eds., Media Technologies. MIT Press, Cambridge, MA, 2014.

[12] Gregson, K. Bad Avatar! Griefing in Virtual Worlds. $A$ Journal of Media and Culture 10, 5 (2007).

[13] Humphreys, S. Ruling the virtual world Governance in massively multiplayer online games. European Journal of Cultural Studies 11, 2 (2008), 149-171.

[14] Humphreys, S. and Zwart, M. de. Griefing, Massacres, Discrimination, and Art: The Limits of Overlapping Rule

Sets in Online Games. UC Irvine Law Review 2, 2 (2012).

[15] Johansson, M. and Verhagen, H. And justice for all - the 10 commandments of online games, and then some... DiGRA Nordic, (2010).

[16] Johansson, M., Verhagen, H., and Kou, Y. I Am Being Watched By The Tribunal-Trust and Control in Multiplayer Online Battle Arena Games. Proceedings of the 10th International Conference on the Foundations of Digital Games, (2015).

[17] Kafai, Y.B. World of Whyville. Games and Culture 5, 1 (2010), 3-22.

[18] Kiesler, S., Siegel, J., and McGuire, T.W. Social psychological aspects of computer-mediated communication. American Psychologist, 39 (1984), 1123-1134.

[19] Kou, Y. and Gui, X. Playing with strangers:

understanding temporary teams in League of Legends.

Proceedings of the first ACM SIGCHI annual symposium on Computer-human interaction in play - CHI PLAY '14, ACM Press (2014), 161-169.

[20] Kou, Y., Gui, X., and Kow, Y.M. Ranking Practices and Distinction in League of Legends. CHI PLAY, (2016).

[21] Kou, Y. and Nardi, B. Regulating Anti-Social Behavior on the Internet: The Example of League of Legends. iConference 2013 Proceedings, (2013), 616-622.

[22] Kou, Y. and Nardi, B. Governance in League of Legends: A Hybrid System. Foundations of Digital Games, (2014).
[23] Kow, Y.M. and Nardi, B. Who owns the Mods? First Monday 15, 5 (2010).

[24] Lin, H. and Sun, C.-T. The "White-Eyed" Player Culture: Grief Play and Construction of Deviance in MMORPGs. DiGRA, (2005).

[25] Lin, J. The Science Behind Shaping Player Behavior in Online Games. Game Developers Conference, (2015).

[26] Lustig, C. and Nardi, B. Algorithmic Authority: The Case of Bitcoin. 2015 48th Hawaii International Conference on System Sciences, IEEE (2015), 743-752.

[27] Matias, J.N. Going Dark: Social Factors in Collective Action Against Platform Operators in the Reddit Blackout. Proceedings of the 2016 CHI Conference on Human Factors in Computing Systems - CHI '16, ACM Press (2016), 11381151.

[28] Merton, R.K. and Robert, N. Contemporary Social Problems. Harcourt Brace, New York, 1966.

[29] Morningstar, C. and Farmer, F.R. The Lessons of Lucasfilm's Habitat. In M. Benedikt, ed., Cyberspace: First Steps. MIT Press, Cambridge, MA, USA, 1991, 273-302.

[30] Phillips, W. LOLing at tragedy: Facebook trolls, memorial pages and resistance to grief online. First Monday 16, 12 (2011).

[31] Ritzer, G. The McDonaldization of Society. The Journal of American Culture 6, 1 (1983), 100-107.

[32] Scott, J.C. and Glaser, B.G. The Discovery of Grounded Theory: Strategies for Qualitative Research. American Sociological Review 36, 1971, 335.

[33] Senior, T. Riot Games hopes tribunal system will clean up League of Legends community. 2011.

http://www.pcgamer.com/2011/01/14/riot-games-hopestribunal-system-will-clean-up-league-of-legendscommunity/.

[34] Suler, J. The online disinhibition effect. Cyberpsychology \& behavior: the impact of the Internet, multimedia and virtual reality on behavior and society 7, 3 (2004), 321-326.

[35] Suzor, N. and Woodford, D. Evaluating Consent and Legitimacy Amongst Shifting Community Norms: an EVE Online Case Study. Journal For Virtual Worlds Research 6, 3 (2013).

[36] Taylor, T.L. Beyond management: Considering participatory design and governance in player culture. First Monday, Special Issue 7 (2006).

[37] Thacker, S. and Griffiths, M.D. An Exploratory Study of Trolling in Online Video Gaming. International Journal of Cyber Behavior, Psychology and Learning 2, 4 (2012), 1733.

[38] Wang, W., Chen, L., Thirunarayan, K., and Sheth, A.P. Cursing in English on twitter. Proceedings of the 17th ACM conference on Computer supported cooperative work \& social computing - CSCW'14, ACM Press (2014), 415-425. [39] Woodford, D. and Darryl, W. Regulating Virtual Worlds: Considering Participant-Driven Approaches. Digital Games Research Association (2013). 